\title{
Predictors of Survival in 211 Patients with Stage IV Pulmonary and Gastroenteropancreatic MIBG-Positive Neuroendocrine Tumors Treated with ${ }^{131}$ I-MIBG
}

\author{
Ari Kane ${ }^{1}$, Matthew P. Thorpe ${ }^{1}$, Michael A. Morse ${ }^{2}$, Brandon A. Howard ${ }^{1}$, Jorge D. Oldan ${ }^{3}$, Jason Zhu ${ }^{2}$, Terence Z. Wong ${ }^{1}$, \\ Neil A. Petry ${ }^{4}$, Robert Reiman, Jr. ${ }^{5}$, and Salvador Borges-Neto ${ }^{1}$ \\ ${ }^{I}$ Department of Radiology, Duke University Medical Center, Durham, North Carolina; ${ }^{2}$ Division of Hematology and Oncology, \\ Department of Medicine, Duke University Medical Center, Durham, North Carolina; ${ }^{3}$ Division of Nuclear Medicine, Department of \\ Radiology, University of North Carolina, Chapel Hill, North Carolina; ${ }^{4}$ Radiopharmacy, Duke University Medical Center, Durham, \\ North Carolina; and ${ }^{5}$ Division of Radiation Safety, Duke University Medical Center, Durham, North Carolina
}

See an invited perspective on this article on page 1706.

This retrospective analysis identifies predictors of survival in a cohort of patients with meta-iodobenzylguanidine (MIBG)-positive stage IV pulmonary and gastroenteropancreatic neuroendocrine tumor (P/GEP$\mathrm{NET})$ treated with ${ }^{131} \mathrm{I}$-MIBG therapy, to inform treatment selection and posttreatment monitoring. Methods: Survival, symptoms, imaging, and biochemical response were extracted via chart review from 211 P/GEP-NET patients treated with ${ }^{131}$ |-MIBG between 1991 and 2014. For patients with CT follow-up $(n=125)$, imaging response was assessed by RECIST 1.1 if images were available $(n=76)$ or by chart review of the radiology report if images could not be reviewed $(n=49)$. Kaplan-Meier analysis and Cox multivariate regression estimated survival and progression-free survival benefits predicted by initial imaging, biochemical response, and symptomatic response. Results: All patients had stage IV disease at the time of treatment. Median survival was 29 mo from the time of treatment. Symptomatic response was seen in $71 \%$ of patients, with the median duration of symptomatic relief being $12 \mathrm{mo}$. Symptomatic response at the first follow-up predicted a survival benefit of $30 \mathrm{mo}(P<0.001)$. Biochemical response at the first clinical follow-up was seen in $34 \%$ of patients, with stability of laboratory values in $48 \%$; response/stability versus progression extended survival by $40 \mathrm{mo}(P<0.03)$. Imaging response (20\% of patients) or stability $(60 \%)$ at the initial 3-mo follow-up imaging extended survival by $32 \mathrm{mo}(P<0.001)$. Additionally, multiple ${ }^{131} \mathrm{I}$-MIBG treatments were associated with 24 mo of additional survival $(P<0.05)$. Conclusion: Therapeutic ${ }^{131}$ I-MIBG for metastatic P/GEPNETs appears to be an effective means of symptom palliation. Imaging, biochemical, and symptomatic follow-up help prognosticate expected survival after ${ }^{131}$ I-MIBG therapy. Multiple rounds of ${ }^{131}$ I-MIBG are associated with prolonged survival.

Key Words: endocrine; radionuclide therapy; MIBG; carcinoid; neuroendocrine tumor; therapy

J Nucl Med 2018; 59:1708-1713

DOI: 10.2967/jnumed.117.202150

\footnotetext{
Received Sep. 12, 2017; revision accepted Apr. 23, 2018.

For correspondence or reprints contact: Salvador Borges-Neto, Duke University Medical Pavilion, 10 Medical Circle, Room 1W19, DUMC Box 3949, Durham, NC 27710.

E-mail: salvador.borgesneto@duke.edu

Published online May 18, 2018.

COPYRIGHT (C) 2018 by the Society of Nuclear Medicine and Molecular Imaging.
}

$\mathbf{T}$ he incidence and prevalence of pulmonary and gastroenteropancreatic neuroendocrine tumor (P/GEP-NET, formerly referred to as carcinoid tumor) has been steadily increasing over the last several decades (1). Neuroendocrine tumors present with metastatic disease in up to $40 \%$ of cases (2). Options for treatment of metastatic disease include somatostatin analogs, targeted therapies, systemic chemotherapy, and locoregional therapies (1). Radiopharmaceuticals have been widely used for imaging and increasingly as therapeutics, most commonly in the form of peptide receptor radiotherapy consisting of a somatostatin receptor binding peptide linked to a chelator bearing the radionuclide of interest. However, $10 \%-20 \%$ of neuroendocrine tumors lack sufficient somatostatin receptor expression for peptide receptor radionuclide therapy (3-5). Further, over the course of the disease, patients frequently exhaust multiple treatment modalities. An alternative is ${ }^{131} \mathrm{I}$-meta-iodobenzylguanidine ( $\left.{ }^{131} \mathrm{I}-\mathrm{MIBG}\right)$, an injectable radiolabeled norepinephrine analog that is taken up by chromaffin cells within neuroendocrine tumors (6). The cells concentrate the radioactive molecule within the neurosecretory granules, where ${ }^{131}$ I emits $\beta$-radiation while sequestered in the tumor cells, offering a molecularly targeted method of in vivo radiation treatment for neuroendocrine tumors.

Prior reports, including one from our group, have provided outcome data after ${ }^{131} \mathrm{I}$-MIBG therapy from small cohorts, short follow-up intervals, or time periods with more limited therapies for neuroendocrine tumors (7-15). Most prior reports also grouped multiple subtypes of neuroendocrine tumors into a single patient group. This current report analyzes our long-term experience with survival and progression-free survival after ${ }^{131} \mathrm{I}-\mathrm{MIBG}$ in 211 patients with metastatic neuroendocrine tumors. Specifically, the goal was to identify predictors of improved overall survival after therapeutic ${ }^{131}$ I-MIBG and how these findings may inform appropriate posttreatment monitoring.

\section{MATERIALS AND METHODS}

This study was performed as a retrospective review of records for consecutive patients referred to Duke University Hospital from 1991 to 2014 for ${ }^{131}$ I-MIBG therapy for metastatic neuroendocrine tumors. The Duke University institutional review board approved this retrospective study, and the requirement to obtain informed consent was waived. Two hundred eleven patients with stage IV P/GEP-NET were identified for further analysis. Chart review extracted relevant clinical 
and demographic data. Survival was determined by chart notation or the Social Security Death Index $(16,17)$. For survival, patients alive at the end of the study period were censored at that time. For progression-free survival, patients were censored at the end of the study period or at the last available follow-up for imaging or symptom assessment.

All patients underwent ${ }^{131}$ I-MIBG or ${ }^{123}$ I-MIBG scanning at our institution before treatment, demonstrating abnormal radiotracer uptake in at least one site of disease. ${ }^{131} \mathrm{I}-\mathrm{MIBG}$ was obtained from the University of Michigan radiopharmaceutical production facility before 2006 and from Nuclear Diagnostic Products after 2006. The total radioactivity in each vial was determined using a radionuclide dose calibrator. Before ${ }^{131}$ I-MIBG administration and for $1 \mathrm{wk}$ after treatment, patients were given either Lugol solution or a saturated solution of potassium iodide to block thyroid accumulation of radioiodine. ${ }^{131} \mathrm{I}-\mathrm{MIBG}$ was administered intravenously by slow infusion over $30-40 \mathrm{~min}$. Per our institutional protocol, 18,500 MBq were administered, with adjustment downward when glomerular filtration rate, blood counts, or liver function tests suggested the patient might be more susceptible to adverse effects. An $18,500-\mathrm{MBq}$ dose was selected on the basis of our empiric practice and assessment of risk-benefit using the estimated critical organ dose with a margin of safety.

${ }^{131}$ I-MIBG therapy was performed on an inpatient basis after discussion of risks and benefits and after the patients had provided written informed consent to the therapy. They were monitored while in radiation isolation until they met the federal regulatory criteria for discharge in effect at the time they were treated (before 1997: a dose rate of $<5 \mathrm{mrem} / \mathrm{h}$ at $1 \mathrm{~m}$ from the patient; after 1997: an expected radiation dose of $<500 \mathrm{mrem}$ to the general public). For the subset of patients referred for repeated ${ }^{131}$ I-MIBG therapy by their oncologists, a repeated diagnostic MIBG scan was performed, and patients with activity in at least one site of metastatic disease received repeated treatment.

$\mathrm{CT}$ and diagnostic MIBG images were reviewed by a radiologist on Centricity PACS (version 2013; GE Healthcare), and response to treatment was assessed using RECIST 1.1. When images were not available for primary review, the dictated reports were used to assess response by noting the appearance of new lesions, the disappearance of lesions, a change in the size of reference lesions, or no change; RECIST 1.1 criteria were applied to given measurements when possible. Patients were categorized as having a complete response, partial response, stable disease, or progressive disease. The initial imaging response was assessed at the first follow-up imaging session, which took place 3-9 mo (median, $3 \mathrm{mo}$ ) after treatment. Symptomatic response was assessed by an oncologist, and patient subjective response was assessed at clinical follow-up visits. Patients who reported no change in symptoms or worsening symptoms were categorized as nonresponders. Laboratory response was assessed when possible, with nonresponders identified by a rise in chromogranin A or urine 5-hydroxyindoleacetic acid characterized by persistence of a greater than $20 \%$ increase from the pretreatment value over 2 serial measurements.

Statistical analysis was performed using SPSS Statistics, version 22.0.0.2 (IBM). Kaplan-Meier survival analysis was performed using the log-rank test for significance with a threshold set to a $P$ value of less than 0.05. Survival statistics were reported using the median survival. Cox regression was used for a subset multivariate analysis of survival.

\section{RESULTS}

Demographic data are shown in Table 1. All patients had stage IV disease at the time of ${ }^{131} \mathrm{I}-\mathrm{MIBG}$ treatment, and $64 \%$ had received prior surgical therapy, radiation therapy, or chemotherapy (Table 2); the clinical charts provided inadequate detail on the number of prior treatments in each category. The median administered activity of ${ }^{131} \mathrm{I}$ MIBG at the first treatment was $18,500 \mathrm{MBq}$ (interquartile range, $11,359-18,870 \mathrm{MBq}$ ), with $19 \%$ of patients receiving 2 administrations
TABLE 1

Demographic Data

\begin{tabular}{ll}
\hline \multicolumn{1}{c}{ Variable } & Data \\
\hline Sex & \\
\hline Male & $50 \%$ \\
\hline Female & $50 \%$ \\
\hline Mean age $(\mathrm{y})$ & $59(\mathrm{SD}, 12)$ \\
\hline Number of treatments & \\
\hline One & $81 \%$ \\
\hline Two & $15 \%$ \\
\hline Three & $3 \%$ \\
\hline Four & $1 \%$ \\
\hline MIBG activity (MBq) & \\
\hline Median & 12,950 \\
\hline Interquartile range & $11,359-18,870$ \\
\hline
\end{tabular}

of ${ }^{131} \mathrm{I}$-MIBG, $4 \%$ receiving 3 administrations, and 1 patient receiving 4 administrations (Table 1). Among those receiving multiple treatments, the median follow-up dose was $12,950 \mathrm{MBq}$ (range, 11,100$18,907 \mathrm{MBq}$ ) and the median total cumulative dose was 33,670 MBq (range, 22,200-37,999 MBq). The median time between the first and second doses was 14 mo (range, 6-25 mo), and that between the second and third doses was 37 mo (range, 14-62 mo). The location of the primary and metastatic lesions, as well as types of ancillary treatments used before and after ${ }^{131} \mathrm{I}-\mathrm{MIBG}$ therapy, is described in Table 2.

Median overall survival from the first ${ }^{131} \mathrm{I}-\mathrm{MIBG}$ treatment was $2.4 \pm 0.2$ y (Fig. 1A). Actual overall 5-y survival from diagnosis was $60 \%$, actual overall 5-y survival from metastatic disease was $50 \%$, and actual overall 5-y survival from eventual treatment with ${ }^{131}$ I-MIBG was $27 \%$. Among patients lost to follow-up, those without follow-up imaging data had a median survival of 14 mo, versus 41 mo in those with follow-up imaging available; those with no biochemical data had a median survival of 23 mo, versus 53 mo in those with follow-up laboratory values available; and those with no symptomatic follow-up had a median survival of 17 mo, versus 39 mo in those with symptomatic follow-up ( $P<0.01$ for each median survival). Multiple ${ }^{131}$ I-MIBG treatments predicted improved survival, compared with patients receiving only one treatment $(4.0 \pm 0.5$ y vs. $2.0 \pm 0.3 \mathrm{y}, P<$ 0.05) (Fig. 2A). Cox regression analysis demonstrated no significant interaction between multiple ${ }^{131}$ I-MIBG treatments, initial treatment response, or use of long-acting octreotide (Sandostatin; Novartis).

One hundred twenty-five patients had CT imaging data at the initial follow-up (median, 3 mo after treatment), of which 76 had images available for primary review and application of RECIST 1.1. Forty-nine patients had radiographic reports for CT follow-up available for review. When images were not available but measurements were provided in reports, RECIST was applied to these measurements $(n=26)$. Thirty-two patients had follow-up MIBG imaging available for review, including 18 patients with MIBG but not CT follow-up. MIBG scans were classified as showing a complete response ( $n=1$, no residual abnormal activity), a partial response ( $n=6$, decreased activity), stable disease $(n=16)$, or disease progression ( $n=9$, increased abnormal activity). Of the 14 patients with both CT and MIBG follow-up, CT and MIBG were concordant in all but 6 patients. Among these 6, 3 showed progression on $\mathrm{CT}$ 
TABLE 2

Sites of Disease and Types of Therapy

\begin{tabular}{|c|c|c|c|c|c|c|c|}
\hline \multicolumn{2}{|c|}{ Primary } & \multicolumn{2}{|c|}{ Metastasis } & \multicolumn{2}{|c|}{ Before ${ }^{131} \mathrm{I}-\mathrm{MIBG}$} & \multicolumn{2}{|c|}{ After ${ }^{131}$ I-MIBG } \\
\hline Site & $n$ & Site & $n$ & Therapy & $\%$ & Therapy & $\%$ \\
\hline Unknown & 83 & Liver & 183 & Surgery & 63 & Chemotherapy & 19 \\
\hline Small bowel & 67 & Bone & 41 & Radiation & 10 & Embolization & 5 \\
\hline Lung & 22 & Lungs & 40 & Embolization & 6 & 131|-MIBG & 19 \\
\hline Colon & 6 & Carcinomatosis & 29 & Chemotherapy & 27 & Surgery & 3 \\
\hline Cecum & 5 & Mediastinum & 17 & Sandostatin & 72 & Radiation & 4 \\
\hline Gastric & 5 & Mesentery & 8 & & & & \\
\hline Rectal & 5 & Adnexa & 6 & & & & \\
\hline Appendix & 4 & Pancreas & 6 & & & & \\
\hline Pancreas & 4 & Adrenal & 4 & & & & \\
\hline Mesentery & 4 & Kidney & 3 & & & & \\
\hline Thymic & 3 & Skin & 2 & & & & \\
\hline Cardiac & 1 & Bladder & 1 & & & & \\
\hline Ovary & 1 & Gastric & 1 & & & & \\
\hline \multirow[t]{4}{*}{ Retroperitoneal } & 1 & Gallbladder & 1 & & & & \\
\hline & & Muscle & 1 & & & & \\
\hline & & Orbit & 1 & & & & \\
\hline & & Spleen & 1 & & & & \\
\hline
\end{tabular}

but stability on MIBG, 1 showed stability on CT and a response on MIBG, and 1 showed stability on CT and progression on MIBG. Patients were considered to have progressed according to imaging if they showed progression on either modality, and they were considered to have responded if they showed a response on either modality. In no case did one modality show response and the other progression.

The initial posttreatment imaging follow-up showed that $80 \%$ of patients were stable or responded $(n=125,2 \%$ complete response, $18 \%$ partial response, $60 \%$ stable disease, $20 \%$ progressive disease), with an imaging-based median progression-free survival of $1.7 \pm$ $0.1 \mathrm{y}(n=143$, Fig. 1C). The median duration of imaging response was 13 mo $(n=143)$. Stability or response at the first imaging follow-up predicted improved survival as compared with radiographic progression $(4.0 \pm 0.4$ y vs. $1.3 \pm 0.3 \mathrm{y}, P<0.001)$ (Fig. $2 \mathrm{~B})$.

The medical charts of 158 patients documented a symptomatic change at the initial posttreatment follow-up. The level of detail across oncology notes was highly variable, allowing only binary assessment of symptomatic response. Seventy-one percent of patients reported an improvement in pretreatment symptoms. The improvement rates were $44 \%$ for pain, $63 \%$ for gastrointestinal complaints (bloating, nausea, vomiting), $43 \%$ for fatigue, $34 \%$ for flushing, and $14 \%$ for unintentional weight loss. Median progression-free survival based on symptoms was $1.4 \pm 0.3 \mathrm{y}$ (Fig. 1B), and the median duration of symptomatic response was 12 mo. A symptomatic response to therapy predicted improved survival $(4.2 \pm 0.4$ y vs. $1.7 \pm 0.6 \mathrm{y}, P<0.001)$ (Fig. $2 \mathrm{C}$ ).

Sixty-two patients had quantitative biochemical data available for review at the initial clinical follow-up: 31 with 5-hydroxyindoleacetic acid, 35 with chromogranin A, and 4 with both. Thirty-four percent of patients demonstrated a response, $48 \%$ were stable, and $18 \%$ progressed (defined as a $20 \%$ increase in 2 consecutive values from baseline). Outcomes did not significantly differ between 5hydroxyindoleacetic acid and chromogranin A. Forty-three percent of patients remained without biochemical progression after treatment throughout the follow-up time, with a median time to biochemical progression of $2.5 \pm 0.5 \mathrm{y}$. Response or stability of chromogranin A or urine 5-hydroxyindoleacetic acid at the initial follow-up predicted improved survival $(6.2 \pm 1.5$ y vs. $2.9 \pm$ $1.6 \mathrm{y}, P<0.03$ ) (Fig. 2D).

\section{DISCUSSION}

Here, we provide outcomes for the largest (to our knowledge) available analysis in the literature of patients with stage IV MIBGpositive P/GEP-NET (formerly known as carcinoid) treated with ${ }^{131}$ I-MIBG. Prior research in this area has primarily comprised small case series (7-14). Overall median survival in our patient group was 29 mo from the time of ${ }^{131}$ I-MIBG treatment. Prior studies have demonstrated median survival of 17-29 mo after treatment $(7,8,14,15)$.

Receiving multiple ${ }^{131} \mathrm{I}-\mathrm{MIBG}$ treatments was associated with 24 mo of additional survival, compared with a single treatment. It is unclear whether this finding represents an additional survival benefit of subsequent therapy or a form of reverse causation by which patients who survive longer are more likely to have time to receive additional therapy. A test of the interaction between receiving multiple treatments and the initial imaging response on follow-up was not significant, suggesting that the initial response did not alter the efficacy of subsequent treatment. The possibility of a benefit from multiple treatments has potential management implications if it can be confirmed in a prospective investigation. In particular, future studies would benefit from more consistent documentation of performance status in the clinical record, as well 


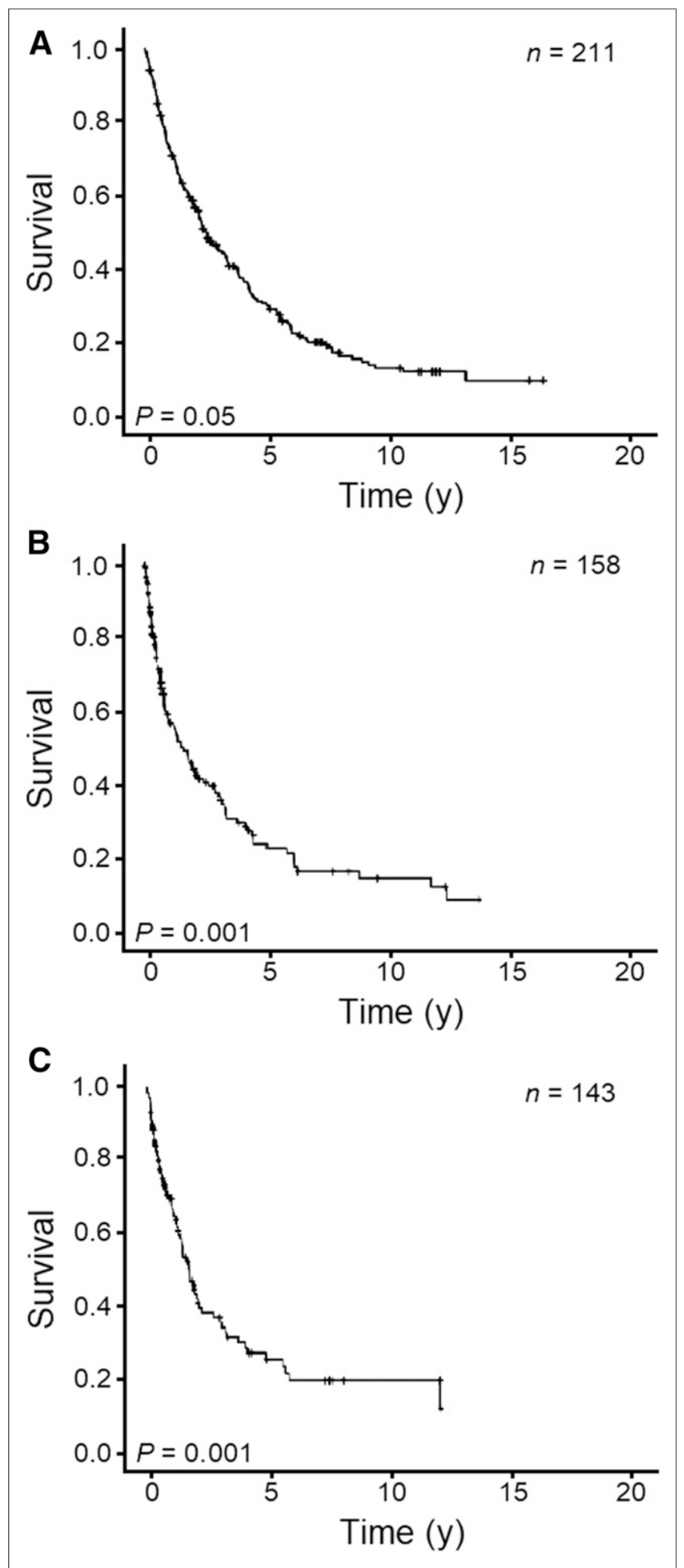

FIGURE 1. Kaplan-Meier curves after ${ }^{131}$ I-MIBG treatment. (A) Overall survival. (B) Symptomatic progression-free survival. (C) Radiographically progression-free survival by CT $(n=125)$ or MIBG $(n=18)$ follow-up.

as the use of some objective index of general wellness to separate the influence of multiple therapies independent of the propensity to survive.

Symptomatic palliation was observed in over $70 \%$ of patients after ${ }^{131}$ I-MIBG therapy; symptomatic improvement lasted a median of $12 \mathrm{mo}$, with a median of 16 mo to subsequent progression. Prior literature reports show a 50\%-90\% symptomatic response rate and a 15 - to 17 -mo median time to progression $(7-15,18)$. Similar to prior studies, symptomatic response predicted improved survival $(11,15)$; in our population, this benefit was 30 mo.

Radiographic response (20\%) and stability (60\%) after ${ }^{131} \mathrm{I}-\mathrm{MIBG}$ treatment were in line with prior studies, which have reported radiographic response rates of $25 \%-35 \%$ and stability rates of $40 \%-50 \%$ $(7,8,11)$. Most prior studies found radiographic response to have no prognostic value $(7,11,15,18)$. In contrast, radiographic response or stability in the current study predicted a 32-mo survival benefit compared with radiographic progression. (Radiographic stability and response were clustered, as their survival benefits did not differ.)

${ }^{131} \mathrm{I}-\mathrm{MIBG}$ treatment yielded a $34 \%$ biochemical response rate and a $48 \%$ stability rate. Prior investigations demonstrated biochemical response rates of $14 \%-55 \%(7,11,14,15)$. No prior studies have shown biochemical response to have a survival benefit-a perplexing finding, particularly given the expected correlation between biochemical abnormalities, symptoms/quality of life, and systemic effects such as cardiac disease. This finding is perhaps in part due to measurement error introduced by heterogeneity in the range of assays used in clinical practice. In our chart review, with many cases predating our electronic record and others relying on the clinical notes of outside providers, insufficient information on the specific assays was available. Despite this additional source of variability, our sample was large enough to demonstrate a 40-mo survival benefit associated with biochemical response/stability, compared with biochemical progression.

Peptide receptor radiotherapy has also demonstrated important survival benefits and symptom palliation. Radiolabeled somatostatin analogs are a more recently developed radioactively labeled peptide group. Prior studies, and the recently reported Neuroendocrine Tumors Therapy (NETTER-1) trial, have demonstrated impressive progression-free survival and symptomatic benefits in midgut neuroendocrine tumors. With peptide receptor radiotherapy, the radiographic response rates of $18 \%-27 \%$ and stability of $60 \%$ are similar to our $20 \%$ response rate and $60 \%$ stability (19-21). Of note, Kwekkeboom et al. reported a decreased responsiveness in GEP-NET (carcinoid) compared with other neuroendocrine tumors (20). These studies found median progression-free survival of 30-32 mo for all patients with neuroendocrine tumors, compared with 21 mo for carcinoid-only patients in the current study (19-21). Only Forrer et al. analyzed symptom response, and they demonstrated good response rates with ${ }^{90}$ Y-DOTATOC for gastrointestinal symptoms and flushing, similar to our study population. They also reported good results in pain reduction, although they did not provide specific numeric outcomes (19). None of the peptide receptor radiotherapy trials have reported comprehensively on biochemical response for comparison to our current study.

Prior studies of chemotherapy demonstrated poor effectiveness for neuroendocrine tumors, with a 3\%-10\% biochemical or imaging response rate and very little symptom palliation $(22,23)$. The median duration of response for chemotherapy was 5 mo $(22,24)$. Octreotide has become a standby for symptomatic neuroendocrine tumor treatment, with high symptom response rates ranging from $30 \%$ to $88 \%$ (25-29). However, octreotide has limited survival benefit, with initial reports from Rinke et al. demonstrating a median time to radiographic progression of 14 mo, versus 6 mo in a placebo group, and the more recent interval release of data from the randomized controlled NETTER-1 trial demonstrating a 


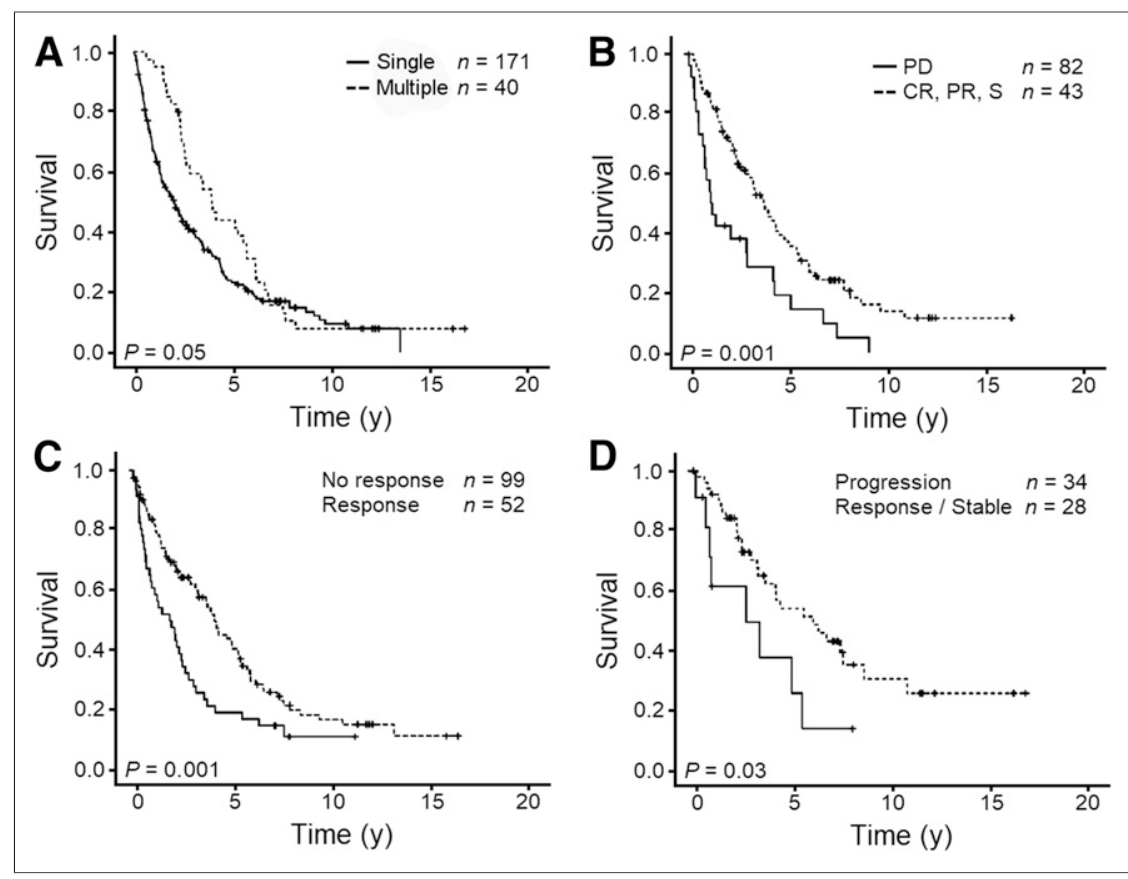

FIGURE 2. Kaplan-Meier survival curves at first follow-up after ${ }^{131}$ I-MIBG treatment. (A) Single vs. multiple ${ }^{131}$ I-MIBG treatment sessions. (B) Imaging demonstrating complete response (CR)/ partial response (PR)/stable disease (S) vs. progressive disease (PD). (C) Symptomatic response vs. no response (or worsening). (D) Biochemical/laboratory response or stability at follow-up vs. progression. $P$ values are for log-rank test.

median time to progression of 8 mo in patients in the octreotide arm $(21,29)$. Patients with hepatic metastatic disease have the option for endovascular embolic treatments, which have demonstrated high efficacy, including response rates of over $70 \%$, a median response duration of $17 \mathrm{mo}$, median progression-free survival of $19 \mathrm{mo}$, and $60 \%$ symptomatic response $(30,31)$.

Because our trial was retrospective and dependent on clinical chart review, many outcomes suffered from missing data. Performance status could not be reliably ascertained from chart review, and we are uncertain whether performance status may interact with the other variables tested. This problem was compounded by the very large catchment area producing referrals to our center for ${ }^{131}$ I-MIBG therapy, as many of these patients were followed up by their local oncologists rather than at our center. Because death dates were available for all participants regardless of clinical followup, overall survival curves are unaffected by the missing data. In contrast, loss to follow-up or incomplete medical records precluding assignment was significant for imaging progression (41\% missing follow-up data), biochemical progression ( $n=28 \%$ missing followup data), and symptomatic progression ( $n=71 \%$ missing follow-up data). Patients with missing data had shortened survival compared with those with complete information, and their loss to follow-up at our center might be explained in part by an overall worsening clinical course. We used customary listwise deletion in managing missing data, finding the remaining sample adequately powered to detect the influence of imaging, symptom, and biochemical response on survival.

\section{CONCLUSION}

We found therapeutic ${ }^{131} \mathrm{I}-\mathrm{MIBG}$ for metastatic P/GEP-NETs to be an effective means of symptom palliation, with improved prognosis for those patients with symptomatic improvement, radiographic response or stability, and biochemical response or stability. This finding suggests that despite previous recommendations (11), regular imaging, biochemical assay, and clinical follow-up should be performed on patients with metastatic neuroendocrine tumors, particularly those undergoing ${ }^{131} \mathrm{I}-\mathrm{MIBG}$ treatment (1). Our results lend tangible data that oncologists can use to provide patients with pretreatment prognostic information, as well as information based on the patient's individual response to treatment. Multiple ${ }^{131}$ I-MIBG treatments were also shown to result in improved survival, suggesting that patients might benefit from multiple rounds of treatment rather than a single treatment session; however, additional research is required to ensure that this association is not confounded by general patient wellness.

Over the course of the disease, many patients with neuroendocrine tumors exhaust the standard options of somatostatin analogs, targeted therapies, and endovascular embolic techniques. Given our findings, ${ }^{131}$ I-MIBG may be of benefit in patients who fail these treatments or whose tumors do not demonstrate the necessary scintigraphic uptake with radiolabeled somatostatin analogs.

\section{DISCLOSURE}

Ari Kane received funding support from the R. Edward Coleman Research Award in Nuclear Medicine and Molecular Imaging. No other potential conflict of interest relevant to this article was reported.

\section{REFERENCES}

1. Maroun J, Kocha W, Kvols L, et al. Guidelines for the diagnosis and management of carcinoid tumours. Part 1: the gastrointestinal tract-a statement from a Canadian National Carcinoid Expert Group. Curr Oncol. 2006;13: 67-76.

2. Quaedvlieg PF, Visser O, Lamers CB, Janssen-Heijen ML, Taal BG. Epidemiology and survival in patients with carcinoid disease in the Netherlands: an epidemiological study with 2391 patients. Ann Oncol. 2001;12:1295-1300.

3. Binderup T, Knigge U, Loft A, et al. Functional imaging of neuroendocrine tumors: a head-to-head comparison of somatostatin receptor scintigraphy, ${ }^{123} \mathrm{I}-$ MIBG scintigraphy, and ${ }^{18}$ F-FDG PET. $J$ Nucl Med. 2010;51:704-712.

4. Intenzo CM, Jabbour S, Lin HC, et al. Scintigraphic imaging of body neuroendocrine tumors. Radiographics. 2007;27:1355-1369.

5. Krenning EP, Kooij PP, Bakker WH, et al. Radiotherapy with a radiolabeled somatostatin analogue, $\left[{ }^{111}\right.$ In-DTPA-D-Phe1]-octreotide: a case history. Ann N Y Acad Sci. 1994;733:496-506.

6. Khan MU, Morse M, Coleman RE. Radioiodinated metaiodobenzylguanidine in the diagnosis and therapy of carcinoid tumors. $Q \mathrm{~J}$ Nucl Med Mol Imaging. 2008;52:441-454.

7. Bomanji JB, Wong W, Gaze MN, et al. Treatment of neuroendocrine tumours in adults with ${ }^{131}$ I-MIBG therapy. Clin Oncol (R Coll Radiol). 2003;15:193-198.

8. Buscombe JR, Cwikla JB, Caplin ME, Hilson AJ. Long-term efficacy of low activity meta- $\left[{ }^{131} I\right]$ iodobenzylguanidine therapy in patients with disseminated neuroendocrine tumours depends on initial response. Nucl Med Commun. 2005;26:969-976.

9. Grünwald F, Ezziddin S. ${ }^{131}$ I-metaiodobenzylguanidine therapy of neuroblastoma and other neuroendocrine tumors. Semin Nucl Med. 2010;40:153-163. 
10. Mukherjee JJ, Kaltsas GA, Islam N, et al. Treatment of metastatic carcinoid tumours, phaeochromocytoma, paraganglioma and medullary carcinoma of the thyroid with ${ }^{131} \mathrm{I}$-meta-iodobenzylguanidine. Clin Endocrinol $(O x f)\left[{ }^{131} \mathrm{I}-\mathrm{mIBG}\right]$. 2001;55:47-60.

11. Nwosu AC, Jones L, Vora J, Poston GJ, Vinjamuri S, Pritchard DM. Assessment of the efficacy and toxicity of ${ }^{131}$ I-metaiodobenzylguanidine therapy for metastatic neuroendocrine tumours. Br J Cancer. 2008;98:1053-1058.

12. Pasieka JL, McEwan AJ, Rorstad O. The palliative role of ${ }^{131} \mathrm{I}-\mathrm{MIBG}$ and ${ }^{111} \mathrm{In}-$ octreotide therapy in patients with metastatic progressive neuroendocrine neoplasms. Surgery. 2004;136:1218-1226.

13. Pathirana AA, Vinjamuri S, Byrne C, Ghaneh P, Vora J, Poston GJ. ${ }^{131}$ I-MIBG radionuclide therapy is safe and cost-effective in the control of symptoms of the carcinoid syndrome. Eur J Surg Oncol. 2001;27:404-408.

14. Taal BG, Hoefnagel CA, Valdes Olmos RA, Boot H, Beijnen JH. Palliative effect of metaiodobenzylguanidine in metastatic carcinoid tumors. J Clin Oncol. 1996;14:1829-1838.

15. Safford SD, Coleman RE, Gockerman JP, et al. Iodine-131 metaiodobenzylguanidine treatment for metastatic carcinoid: results in 98 patients. Cancer. 2004; 101:1987-1993.

16. United States Social Security death index. FamilySearch website. https://familysearch.org/search/collection/1202535. Accessed August 2, 2018.

17. U.S., Social Security Death Index, 1935-2014. Ancestry website. http://search. ancestry.com/search/db.aspx $? \mathrm{dbid}=3693 \& \mathrm{cj}=1 \&$ netid $=$ cj\&o_xid $=0000584978 \&$ o_lid=0000584978\&o_sch=Affiliate+External. Accessed August 2, 2018.

18. Sywak MS, Pasieka JL, McEwan A, Kline G, Rorstad O. ${ }^{131}$ I-meta-iodobenzylguanidine in the management of metastatic midgut carcinoid tumors. World $J$ Surg. 2004;28:1157-1162.

19. Forrer F, Waldherr C, Maecke HR, Mueller-Brand J. Targeted radionuclide therapy with ${ }^{90}$ Y-DOTATOC in patients with neuroendocrine tumors. Anticancer Res. 2006;26:703-707.

20. Kwekkeboom DJ, de Herder WW, Kam BL, et al. Treatment with the radiolabeled somatostatin analog $\left[{ }^{177} \mathrm{Lu}-\mathrm{DOTA}{ }^{0}, \mathrm{Tyr}^{3}\right]$ octreotate: toxicity, efficacy, and survival. J Clin Oncol. 2008;26:2124-2130.

21. Strosberg JR, Wolin EM, Chasen B, et al. NETTER-1 phase III: progression-free survival, radiographic response, and preliminary overall survival results in patients with midgut neuroendocrine tumors treated with 177-Lu-Dotatate [abstract]. J Clin Oncol. 2016;4(suppl):194.

22. Di Bartolomeo M, Bajetta E, Bochicchio AM, et al. A phase II trial of dacarbazine, fluorouracil and epirubicin in patients with neuroendocrine tumours-a study by the Italian Trials in Medical Oncology (I.T.M.O.) Group. Ann Oncol. 1995;6:77-79.

23. Ollivier S, Fonck M, Becouarn Y, Brunet R. Dacarbazine, fluorouracil, and leucovorin in patients with advanced neuroendocrine tumors: a phase II trial. Am J Clin Oncol. 1998;21:237-240.

24. Dahan L, Bonnetain F, Rougier P, et al. Phase III trial of chemotherapy using 5fluorouracil and streptozotocin compared with interferon alpha for advanced carcinoid tumors: FNCLCC-FFCD 9710. Endocr Relat Cancer. 2009;16:1351-1361.

25. de Herder WW, Hofland LJ, van der Lely AJ, Lamberts SW. Somatostatin receptors in gastroentero-pancreatic neuroendocrine tumours. Endocr Relat Cancer. 2003;10:451-458.

26. Faiss S, Pape UF, Bohmig M, et al. Prospective, randomized, multicenter trial on the antiproliferative effect of lanreotide, interferon alfa, and their combination for therapy of metastatic neuroendocrine gastroenteropancreatic tumors: the International Lanreotide and Interferon Alfa Study Group. J Clin Oncol. 2003;21: 2689-2696.

27. Kvols LK, Moertel CG, O’Connell MJ, Schutt AJ, Rubin J, Hahn RG. Treatment of the malignant carcinoid syndrome: evaluation of a long-acting somatostatin analogue. N Engl J Med. 1986;315:663-666.

28. Lamberts SW, van der Lely AJ, de Herder WW, Hofland LJ. Octreotide. N Engl J Med. 1996;334:246-254.

29. Rinke A, Muller HH, Schade-Brittinger C, et al. Placebo-controlled, double-blind, prospective, randomized study on the effect of octreotide LAR in the control of tumor growth in patients with metastatic neuroendocrine midgut tumors: a report from the PROMID Study Group. J Clin Oncol. 2009;27:4656-4663.

30. Gupta S, Yao JC, Ahrar K, et al. Hepatic artery embolization and chemoembolization for treatment of patients with metastatic carcinoid tumors: the M.D. Anderson experience. Cancer J. 2003;9:261-267.

31. Yao KA, Talamonti MS, Nemcek A, et al. Indications and results of liver resection and hepatic chemoembolization for metastatic gastrointestinal neuroendocrine tumors. Surgery. 2001;130:677-682. 